kill cells and inactivate enzymes to which it has been fused. Such innovations mark the rise of "a principally novel type of fluorescent protein," says Lukyanov, "that can be used as active light-operated 'manipulators' rather than passive reporters."

1. Chalfie, M., Tu, Y., Euskirchen, G., Ward, W.W. \& Prasher, D.C. Green fluorescent protein as a marker for gene expression. Science 263, 802-805 (1994).

2. Crameri, A., Whitehorn, E.A., Tate, E. \& Stemmer, W.P. Improved green fluorescent protein by molecular evolution using DNA shuffling. Nat. Biotechnol. 14 , 315-319 (1996)

3. Yang, F., Moss, L.G. \& Phillips, G.N. Jr. The molec ular structure of green fluorescent protein. Nat. Biotechnol. 14, 1246-1251 (1996).

4. Matz, M.V. et al. Fluorescent proteins from nonbio luminescent Anthozoa species. Nat. Biotechnol. 17, 969-973 (1999).

5. Nagai, T. et al. A variant of yellow fluorescent protein with fast and efficient maturation for cell-biological applications. Nat. Biotechnol. 20, 87-90 (1999).

6. Bevis, B.J. \& Glick, B.S. Rapidly maturing variants of the Discosoma red fluorescent protein (DsRed). Nat. Biotechnol. 20, 83-87 (2002).

7. Chudakov, D.M. et al. Photoswitchable cyan fluorescent protein for protein tracking. Nat. Biotechnol. 22, 1435-1439 (2004).

8. Bulina, M.E. et al. A genetically encoded photosen sitizer. Nat. Biotechnol. 24, 95-99 (2006).

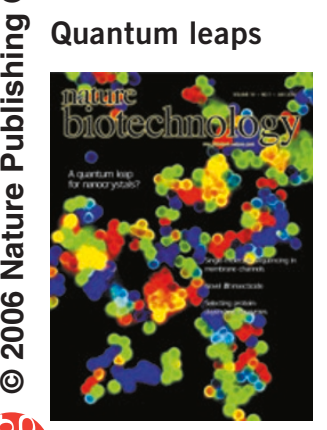

Quantum dots have properties imagers long for. The nanocrystals absorb a broad spectrum of light, but the light they emit can be tuned to any narrow frequency. Compared to organic dyes, they can be 20 times brighter and orders of magnitude more stable to photobleaching. But at several nanometers across, the dots are closer in size to proteins than small molecules; a quality that could limit their uses in cells. In 2003, researchers at Quantum Dot (Hayward, CA, USA) showed that dots could label cancer markers, actin and nuclear antigens inside cancer cells ${ }^{1}$. The same year, researchers at Rockefeller University (New York) showed that antibodies could be conjugated to quantum dots, and that cells taking in the conjugates grew for over a week ${ }^{2}$. In 2004, in what one commentator hailed as a possible precursor to an 'optical biopsy', a team led by Shuming Nie, director of the Emory-Georgia Tech Nanotechnology Center (Atlanta, GA, USA) demonstrated for the first time that quantum dots could be used to image cancer in living animals ${ }^{3}$. To do so, Nie and his team encased the quantum dots in an amphiphilic triblock shell. This allows multiple functionalities to be attached to each dot, and provides a general platform to add molecules that target tumors, enhance permeability or bestow other properties. In nude mice with human prostate cancer xenografts, the dots accumulated in tumors, allowing sensitive and multicolor fluorescence

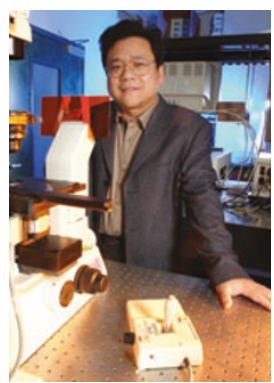

Shuming Nie: "We want something that is clinically realistic."

imaging.

Others also started to explore the applications of quantum dots in imaging. In perhaps the first paper to apply quantum dots to a surgical procedure, Harvard oncologist John Frangioni showed that quantum dots could transform a difficult, error-prone surgery used to detect cancer spread ${ }^{4}$ - sentinel lymph node biopsy-into a straightforward procedure that no longer requires radioactive tracers. Key to this was the design of quantum dots that emit near-infrared light, which is scattered less by tissues than is visible light. Combined with an imaging system that integrated visible and near-infrared light, the dots clearly identified sentinel lymph nodes as deep as $1 \mathrm{~cm}$ below the skin in a living, $35-\mathrm{kg}$ pig, allowing the nodes to be removed with minimal dissection.

But Frangioni doesn't expect quantum dots to guide human surgery anytime soon, if ever. Instead, he hopes to replace the dots with albumin attached to indocyanine green, a near infrared fluorescent dye. These probes won't work as well as the quantum dots, which can be engineered to a size that will lodge in the sentinel nodes, but that's not the point. "Now that we've proved principle," he says, "we want something that's clinically realistic."

Frangioni thinks near-infrared systems will eventually image tissues as deep as 4 $\mathrm{cm}$ below the skin, but that penetrance will depend not on fluorophores, but on detection systems. For quantum dots, he says, the most significant barrier is potential toxicity. Dots are typically made from heavy metals. They are too big to be removed by the kidney and too foreign to be metabolized by the liver, so they remain in the body indefinitely. To create a formulation suitable for humans, Frangioni and Massachusetts Institute of Technology collaborator Moungi Bawendi are developing smaller dots made from different elements.

Over the next five to ten years, both Frangioni and Nie believe quantum dots have the greatest potential for in vitro biomedical applications, particularly more sensitive detection of biomarkers. In fact, Nie's group has already developed microbeads encrusted with biodetecting quantum dots emitting different colors and intensities ${ }^{5}$. Theoretically, these beads could generate millions of distinct colors, a capacity beyond any imaginable practical applications. So far, about 20 different types can be detected at once, though Nie thinks the ultimate number could be as high as a thousand. The technology has already been commercialized by Crystalplex (Pittsburgh, PA, USA). Nie thinks issues of penetration and toxicity will likely keep in vivo applications at bay for several years, but he's not giving up. In fact, he is already putting his coatings onto nearinfrared quantum dots like the ones designed by Frangioni.

1. Wu, X. et al. Immunofluorescent labeling of cancer marker Her2 and other cellular targets with semiconductor quantum dots. Nat. Biotechnol. 22, 41-46 (2003).

2. Jaiswal, J.K. et al. Long-term multiple color imaging of live cells using quantum dot bioconjugates. Nat Biotechnol. 21, 47-51 (2003)

3. Gao X. et al. In vivo cancer targeting and imaging with semiconductor quantum dots. Nat. Biotechnol. 22, 969-976 (2004).

4. Kim S. et al. Near-infrared fluorescent type II quantum dots for sentinel lymph node mapping. Nat. Biotechnol. 22, 93-97 (2004)

5. Han, M., Gao, X., Su, J.Z. \& Nie, S. Quantum-dottagged microbeads for multiplexed optical coding of biomolecules. Nat. Biotechnol. 19, 631-635 (2001)

\section{The delivery problem}

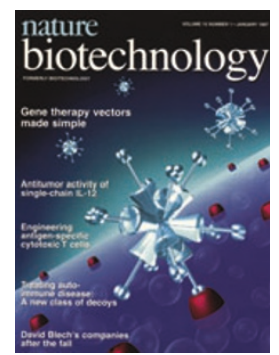

Delivering drugs, nucleic acids and proteins into cells in a safe and efficient manner has been, and remains, one of the most challenging problems in biotech. Robert Debs still wants to get DNA into cells, but he's largely through fiddling with cationic liposomes. In 1997, Debs, of San Francisco's California Pacific Medical Center Research Institute, reported that big, multilayered vesicles studded with cholesterol could improve the efficiency of gene delivery in living animals by as much as 1,740 -fold ${ }^{1}$. A few months later, Nancy Templeton and her colleagues, at Baylor College of Medicine in Austin, Texas, showed how processes like sonication, heating and optimizing DNA/liposome ratios could boost systemic gene delivery ${ }^{2}$. Debs and colleagues were subsequently able to increase efficiency 